\title{
Review: smooth muscle relaxants treat abdominal pain and loperamide reduces diarrhoea in irritable bowel syndrome
}

\author{
Jailwala J, Imperiale TF, Kroenke K. Pharmacologic treatment of the irritable bowel syndrome: a systematic review of \\ randomized, controlled trials. Ann Intern Med 2000 Jul 18;133:136-47.
}

\section{QUESTION: What is the effectiveness of pharmacological agents for the treatment of irritable bowel syndrome (IBS)?}

\section{Data sources}

Studies were identified by searching Medline (1966-99), EMBASE/Excerpta Medica (1980-99), PsycINFO (1967-99), and the Cochrane Controlled Trials Registry with terms that included colonic diseases, functional, irritable, spastic, bowel, and colon and by manually searching bibliographies of relevant studies.

\section{Study selection}

Published studies in the English language were selected if they examined use of a pharmacological treatment for IBS on $>10$ adult patients for $\geqslant 2$ weeks; included a placebo group; reported an outcome measure of global status or individual symptoms, or both, of IBS; and used a randomised, double blind, parallel group or crossover design.

\section{Data extraction}

Data were extracted on the diagnostic criteria for IBS, participant characteristics, interventions, study design, methodological quality (scored between 1 and 5 with a higher score representing higher quality; studies with a score $\geqslant 4$ were classified as "high quality"), and outcomes. Pharmacological agents were classified as having "positive" effectiveness if the study reported significant improvement in global status or individual IBS symptoms; otherwise, they were classified as having "negative" effectiveness.

\section{Main results}

70 studies (4836 patients, median age 38 y, $68 \%$ women, mean study duration 7.5 wks, mean methodological quality score 3.2 ) met the inclusion criteria. 66 studies evaluated a single agent, and 4 evaluated a combination of $\geqslant 2$ agents. The most common pharmacological classes of agents were smooth muscle relaxants, bulking agents, prokinetic agents, psychotropic agents, and loperamide. Of 16 studies of smooth muscle relaxants, 13 showed positive effectiveness; 7 had high quality, and all of them showed an improvement in abdominal pain. Of 13 studies of bulking agents, 4 showed positive effectiveness; 7 had high quality, and of these, 3 showed positive effectiveness. Of 6 studies of prokinetic agents, 2 showed positive effectiveness; 4 had high quality, and of these, 1 showed positive effectiveness. All 7 studies of psychotropic agents showed positive effectiveness, and 1 had high quality. All 4 studies of loperamide showed an improvement in diarrhoea, and 2 had high quality.

\section{Conclusions}

Most studies on pharmacological treatment of irritable bowel syndrome examined smooth muscle relaxants and bulking agents. Smooth muscle relaxants are effective for relieving abdominal pain, and loperamide is effective for reducing diarrhoea. However, data are inconclusive for the effectiveness of other pharmacological agents for irritable bowel syndrome.
Source of funding: not stated.

For correspondence: Dr T F Imperiale, Health Services

Research and Development, Route 11-H,Roudebush Veterans Affairs Medical Center, 1481 West 10th Street, Indianapolis, IN 46202, USA. Fax +1 3175540114.

\section{COMMENTARY}

IBS is still a diagnosis of exclusion despite the fact that criteria for making a positive diagnosis have been established and widely disseminated. Organic lesions that resemble symptoms commonly seen in IBS must be excluded before initiating treatment. ${ }^{1}$ The traditional treatment for IBS has been a combination of symptomatic therapy, reassurance, education, and lifestyle modification. Patients with milder disease can usually be managed conservatively, even for the short term. Other patients can be prescribed a combination of psychotherapy, psychopharmacotherapy, and pharmacotherapy, which generally has variable success.

The review by Jailwala $e t$ al evaluates the available therapeutic trials. Considering the varied presentation of IBS, lack of uniform diagnostic criteria, and initial difficulties in defining the condition, a substantial number of trials did not meet rigorous scientific standards. The authors stated that smooth muscle relaxants for abdominal pain and loperamide for diarrhoea were the only agents with clinical effectiveness. Although results for other therapeutic agents were inconclusive, the authors expressed an optimistic view that, as the pathophysiology of IBS becomes better understood, it is likely that subgroups of patients with specific sets of symptoms may be responsive to targeted treatments.

Many new treatments that focus on modulating neurotransmitters in the "brain-gut" axis are currently being studied. Recent interest in serotonin receptors and serotonin mediated motor activity in the gut has led to the development of new treatment options for the management of the more distressing symptoms associated with IBS. The type 3 serotonin receptor $\left(5-\mathrm{HT}_{3}\right)$ stimulates extrinsic enteric sensory nerves, thereby lowering visceral pain thresholds, a generally accepted component of IBS. ${ }^{2}$

The study by Camilleri $e t$ al involved an impressive number of women who had abdominal pain and principally diarrhoea predominant IBS. The authors were able to show reasonable, although not overwhelmingly impressive, therapeutic efficacy of the $5-\mathrm{HT}_{3}$ antagonist alosetron for control of mild to moderate symptoms.

Alosetron (Lotronex) is the first serotonin subtype antagonist to be examined in a large clinical trial. Unfortunately, alosetron's adverse effects have led to its recent voluntary withdrawal by its manufacturer, Glaxo Wellcome. This decision followed discussions 